\title{
Welche Anreizwirkungen hat der Morbi-RSA hinsicht- lich des Leistungs- und Qualitätsmanagements bei den Krankenkassen und Leistungserbringern?
}

Mit Inkrafttreten des Gesetzes zur Stärkung des Wettbewerbs in der gesetzlichen Krankenversicherung (GKV-WSG) am 1. April 2007 hat der Gesetzgeber die Weichen zu einer Weiterentwicklung des Risikostrukturausgleichs (RSA) gestellt. Damit sollte dieser in seiner Funktion gestärkt werden, die finanziellen Auswirkungen von Risikoselektion zu neutralisieren. Hiervon verspricht man sich, die Preissignalwirkung des Beitragssatzes besser gewährleisten und eine verstärkte Ausrichtung des Kassenwettbewerbs auf Qualität und Wirtschaftlichkeit ermöglichen zu können. Die bis dato allein auf den Ausgleichsfaktoren Alter, Geschlecht, Bezug/Nicht-Bezug einer Erwerbsminderungsrente sowie Teilnahme an einem DMP (seit 2002) beruhende Ermittlung und kassenspezifische Zurechnung von Beitragsbedarfen war in diesem Zusammenhang als zu ungenau und unfair erachtet worden. In seiner neuen Form soll das - dann stärker morbiditätsorientierte - Ausgleichsverfahren ab dem 1. Januar 2009 Anwendung finden und zu wesentlich zielgenaueren Mittelzuweisungen führen. Das Klassifikationsmodell wird aber nicht das gesamte Krankheitsspektrum der Versicherten berücksichtigen, sondern lediglich 80 Krankheiten, die „insbesondere kostenintensiv-chronischer Natur sind oder einen schwerwiegenden Verlauf aufweisen“. Durch die Kostenneutralität werden die weiterhin an die Kassen allozierten Zuweisungen nach Alter und Geschlecht wesentlich geringer als heute ausfallen, da der Rest der Verteilsumme auf die noch nicht quantifizierten Krankheitszuschläge entfällt.

Dadurch ergeben sich drei Gruppen von Versicherten: (1) mit zuschlagsrelevanten Krankheiten, (2) mit Krankheiten, die nicht zuschlagsrelevant sind und (3) „Gesunde“. Kritiker befürchten, dass Kassen bei einem so gestalteten morbiditätsorientierten RSA dazu verleitet sein könnten, das Ziel der Minimierung von Leistungsausgaben über Effizienzverbesserungen dem Ziel der Maximierung von Transfers aus dem RSA unterzuordnen. Indem sie ihr jeweiliges Versichertenkollektiv möglichst krank und behandlungsbedürftig erscheinen ließen und dieses in zusätzliche oder monetär höher bewertete Risikokategorien brächten, könnten die Kassen den ihnen zurechenbaren Beitragsbedarf im RSA erhöhen. Am unattraktivsten für die Krankenkassen werden Versicherte der Gruppe 2 sein; der Anreiz, diesen Merkmale zuzuordnen, die zu einer Zuordnung in Gruppe 1 führt, wird groß sein - insbesondere wenn „nur“ bestimmte Medikamente zur Eingruppierung fehlen (z.B. wenn der insulinpflichtige Diabetes vom BVA über die Insulin-Verschreibung „,validiert" wird [1]). Es könnte allerdings auch so sein, dass präventive Bemühungen konterkariert werden, wenn sie „erfolgreich“ die Entwicklung einer zuschlagsberechtigten Erkrankung verhindern.

Eine solche „Morbidisierung“ von Versichertenkollektiven könnte entweder aktiv über eine entsprechende Steuerung und Einflussnahme auf das Versorgungsgeschehen erfolgen; oder sie geschähe passiv über ein Nachlassen der kostspieligen Bemühungen um (a) Dokumentationsqualität („up-coding“), (b) prozessorientierte Leistungssteuerung etwa über Leitlinien („gaming“, d.h. unnötige Leistungen werden tatsächlich erbracht) und (c) ergebnisorientierte Qualitätsverbesserungen auf der Leistungsseite und damit (d) Begrenzung des Ausgabenanstiegs (in diesem Zusammenhang sei erwähnt, dass das BVA entgegen dem Gutachtervorschlag [2] auch stationäre Komplikationen als Krankheiten definiert hat, die für die Kassen zu einem Zuschlag führen). Dass künftig gleichzeitig auch die Vergütung von Leistungserbringern an ähnlich aus dem Versorgungsgeschehen abgeleiteten Morbiditätsindikatoren (G-DRGs, diagnosebezogene Regelleistungsvolumina) bemessen wird, wird als problemverschärfend gesehen: Gleichgerichtete Interessen von Kostenträgern und Leistungserbringern würden als Kostentreiber fungieren. Bei den Versicherten in Gruppe 1 besteht die Gefahr, dass auch hier nicht Qualität und Angemessenheit der Behandlung im Mittelpunkt stehen, sondern das Aufrechterhalten der zur Eingruppierung führenden Kriterien (so wäre es kontraproduktiv, einen Diabetiker erfolgreich mit einem Ernährungsprogramm zu behandeln, wenn Antidiabetika zur Eingruppierung notwendig sind). Da gegenüber der heutigen Rechtslage die Einschreibung in DMPs nicht mehr zu einer getrennten Berücksichtigung im RSA führen wird, werden sich auch hier gravierende Anreizverschiebungen ergeben: DMPs lohnen sich nur noch, wenn sie nachweislich zu Kostensenkungen führen.

Autorenerklärung: Es bestehen keine finanziellen Interessenkonflikte in Zusammenhang mit diesem Manuskript.

\section{Literatur}

1 Behrend $C$, Felder $S$, Busse $R$. Zur Strategieanfälligkeit der Arzneimittelkomponente des IPHCC+RXGroups-Klassifikationssystems in einem morbiditätsorientierten Risikostrukturausgleich - Eine konzeptionelle und datengestützte Analyse. Gesundheitswesen 2007; 69 (1): 1-10

2 Busse R, Drösler S, Glaeske G, Greiner W, Schäfer T, Schrappe M (Wissenschaftlicher Beirat zur Weiterentwicklung des Risikostrukturausgleichs). Wissenschaftliches Gutachten für die Auswahl von 50 bis 80 Krankheiten zur Berücksichtigung im morbiditätsorientierten Risikostrukturausgleich. Bonn: 2007
R. Busse

Qualitätsmanagement

Schlüsselwörter

Anreizwirkungen

Mittelverteilung

Über-/Unterversorgung

Key words

Oincentives

financial allocation

over-/undertreatment

Institut

Fachgebiet Management im Gesundheitswesen, Technische Universität Berlin

Bibliografie

DOI 10.1055/s-0028-1085586

Dtsch Med Wochenschr 2008:

133: S139 - (c) Georg Thieme

Verlag KG Stuttgart · New York . ISSN 0012-0472

Korrespondenz

Prof. Dr. med. Reinhard Busse Fachgebiet Management im Gesundheitswesen

Technische Universität Berlin, Sekr. $\mathrm{H} 80$

Str. des 17. Juni 135

10623 Berlin

Tel. +49 (30) 314-28420

eMailmig@tu-berlin.de 